\title{
Adult Bochdalek hernia complicated with a perforated colon
}

\author{
Ying Chai, MD, Guofei Zhang, MD, and Gang Shen, MD, Hangzhou, China
}

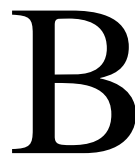

ochdalek hernia usually presents in the neonatal period but rarely presents in adulthood. The diagnosis of this disease is not always easy, as is demonstrated in our case. In this study we describe a case of adult Bochdalek hernia complicated with a perforated colon, which was successfully treated by means of video-assisted thoracic surgery (VATS) and laparotomy.

\section{Clinical Summary}

In 2005, a 46-year-old woman presented to a local hospital with a 15-day history of unremitting left upper abdominal dull pain. Moreover, the patient had vomited profusely and could not keep down anything for 5 days. On physical examination, the abdomen was soft, with no signs of peritoneal irritation, but reduced breath sounds in the left lower chest were noticed. The chest radiograph (Figure 1) on hospital admission revealed pleural fluid and gas with compression of the left basal lung, causing suspicion of pleural empyema. Then left closed chest drainage was performed. The patient's condition deteriorated on chest radiography (Figure 2) on the second day, which showed an increased area of fluid and air in the left thorax, with mediastinal displacement to the right. The patient reported having respiratory distress.

The patient was transferred to our care center for further management at that time. The liquid from the chest tube looked like something that was ingested by mouth, and it caused the suspicion of rupture of the esophagus with perforation to the pleural cavity. However, the upper gastrointestinal tract barium meal did not provide any new information. Then a left VATS was performed, the compressed left lung was covered with necrotized fibrinoid tissue, and air and pus were seen in the pleural cavity. A 5-cm long posterolateral diaphragmatic defect was identified, with the colon herniating through the opening, and the colon was perforated by the chest tube with a hole of $1.0 \times 1.0 \mathrm{~cm}$. At that time, the diagnosis of Bochdalek hernia was made. After lysis of adhesions between the colon and the pleural cavity and exfoliation of the necrotized fibrinoid tissue from the lung surface, the left compressed lung was fully expanded. Then, through an abdominal incision, the herniated colon bowel was put back gently into the peritoneal cavity, and a colectomy with an end-to-end anastomosis

From the Department of Thoracic Surgery, 2nd Affiliated Hospital, School of Medicine, Zhejiang University, Hangzhou, China.

Received for publication July 11, 2005; accepted for publication Aug 8, 2005 .

Address for reprints: Ying Chai, MD, 88 Jiefang Road, Hangzhou, 310009, China (E-mail: chai_y@126.com).

J Thorac Cardiovasc Surg 2005;130:1729-30

$0022-5223 / \$ 30.00$

Copyright $\odot 2005$ by The American Association for Thoracic Surgery

doi:10.1016/j.jtcvs.2005.08.032

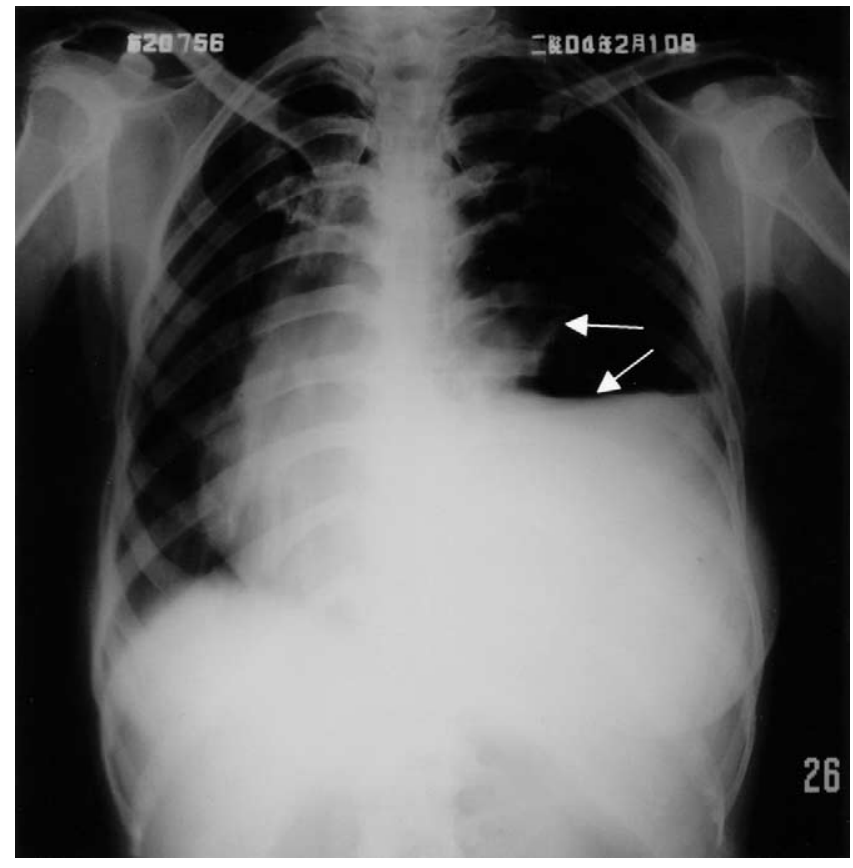

Figure 1. The anteroposterior chest radiograph before performing closed chest drainage, revealing gas, liquid, and an air-fluid level (arrow), with the compression of the left basal lung (arrow).

was performed to remove the suffering colon. The diaphragmatic defect was closed with interrupted nonabsorbable sutures.

Recovery after the operation went well and was without complications. The subsequent chest radiograph showed the left lung fully expanded, including the site of previous hernia. Six months after discharge, the patient was still well.

\section{Discussion}

The Bochdalek foramen is located between the costal part and the lumbar part of the diaphragm. Normally, this area contains muscle, but in these patients the fusion of the 2 parts is disordered during embryogenesis, retaining a posterolateral defect or only a phrenoperitoneal fold. For any increase in intra-abdominal pressure, such as labor, pregnancy, and trauma, the abdominal viscera can be herniated into the thorax through the defect, causing Bochdalek hernia. $^{1}$

Bochdalek hernia in adulthood presents with nonspecific characteristics, such as chronic dyspnea, recurrent pain, nausea, and vomitus, ${ }^{1,2}$ and therefore it often leads to a difficult diagnosis, as in our case. If adult patients present with acute problems, they always coexist with intestinal obstruction or hollow organ perforation. However, some patients are asymptomatic, and their conditions are detected at routine chest radiography. ${ }^{2}$ 


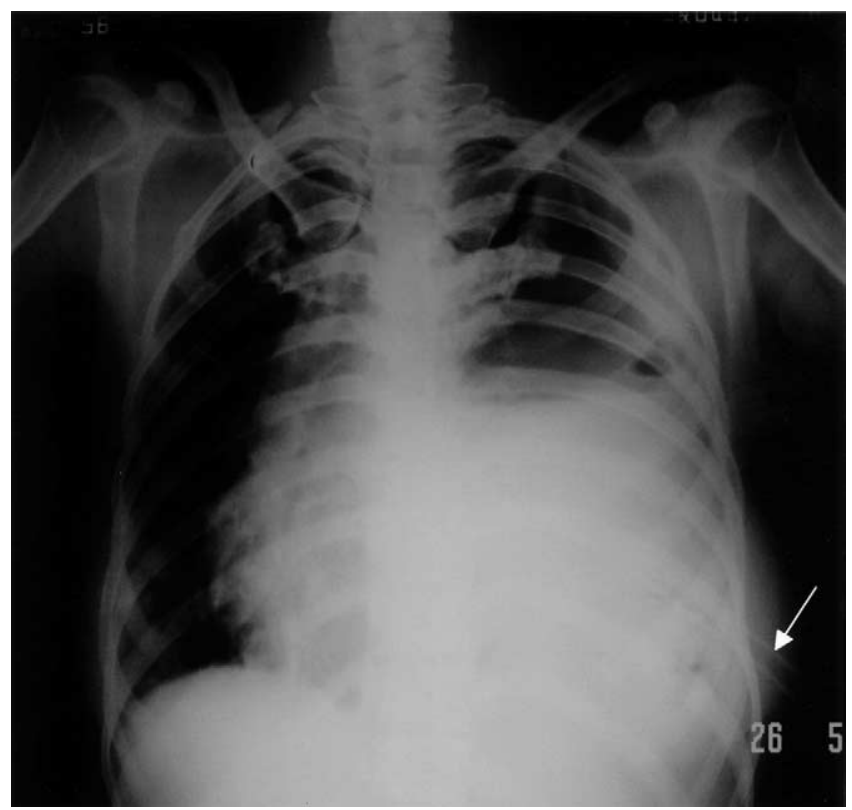

Figure 2. Chest radiograph after closed chest drainage (arrow), showing an increased area of fluid and air in the left thorax, with mediastinal displacement to the right.

Radiography is adequate to diagnose large herniations, but most small ones are not visible, which can be confirmed by means of computed tomography or magnetic resonance imaging. ${ }^{2,3}$ These reveal gas and liquid in the lung fields, presenting as shadowing by the herniated parenchymal organs. Appropriate contrast studies of the gastrointestinal tract, such as with a barium meal or barium enema, will demonstrate herniation in many cases. ${ }^{1}$ However, its abnormal signs do not exclude the diagnosis of herniation. Misdiagnosis of this disease can take place on the basis of the signs of radiology, such as pneumothorax and hemothorax. In these pa- tients unnecessary procedures, like chest tube drainage, should be avoided.

If the patients have chronic or acute respiratory and digestive problems, they require rapid surgical intervention. For asymptomatic patients, because sudden deterioration can happen, such as strangulation or incarceration of hernial parts, we suggest they should undergo accepted repair at the proper time. Most groups favor thoracotomy for right-sided defects and advocate laparotomy or thoracotomy for the left side. ${ }^{4}$ Sometimes because of the proximity of the lesion to the chest wall, pleuroperitoneal adhesions, or the perforation of the hernia viscera, repair is more difficult through the transthoracic approach, and then an additional abdominal approach is needed. The viscera should be gently reduced to the abdomen. Inspection of the viscera should show no evidence of ischemic damage, or the suffering viscera should be resected. Most defects are repaired primarily, except for a large defect, which required a polytetrafluoroethylene patch (Gore-Tex patch; W. L. Gore \& Associates, Inc, Flagstaff, Ariz). ${ }^{5}$ The other associated abnormalities should be treated at the same time. We suggest that repair of a nonincarcerated Bochdalek hernia by way of VATS is a better approach. In our case we repaired the hernia using left VATS and laparotomy, and postoperative recovery was uneventful.

\section{References}

1. Perch P, Houck WV, DeAnda AJ. Symptomatic Bochdalek hernia in an octogenarian. Ann Thorac Surg. 2002;73:1288-9.

2. Uchino A, Yoshida N, Ohnari N, Ohno M. Asymptomatic Bochdalek hernia diagnosed by magnetic resonance imaging. Radiat Med. 1990; 8:58-60.

3. Shin MS, Mullingan SA, Baxley WA, Ho KJ. Bochdalek hernia of diaphragm in the adult diagnosis by computed tomography. Chest. 1987;92: 1098-101.

4. Yamaguchi M, Kuwano H, Hashizume M, Sugio K, Sugimachi K, Hyoudou Y. Thoracoscopic treatment of Bochdalek hernia in the adult: report of a case. Ann Thorac Cardiovasc Surg. 2002;8:106-8.

5. Bruscinano L, Izzo G, Maffettone V, Rossetti G, Renzi A, Napolitano V, et al. Laparoscopic treatment of Bochdalek hernia without the use of a mesh. Surg Endosc. 2003;17:1497-8. 